Supporting information for

\title{
Synthesis, characterization, and enthalpies of formation of uranium substituted zirconolites
}

Tamilarasan Subramani ${ }^{1}$, Jason Baker ${ }^{2}$, Hongwu $X u^{2}$ and Alexandra Navrotsky ${ }^{1}$

${ }^{1}$ School of Molecular Sciences and Center for Materials of the Universe, Arizona State University,

Tempe, Arizona, 85287, USA

${ }^{2}$ Earth and Environmental Sciences Division, Los Alamos National Laboratory, Los Alamos, New Mexico, 87545, USA 

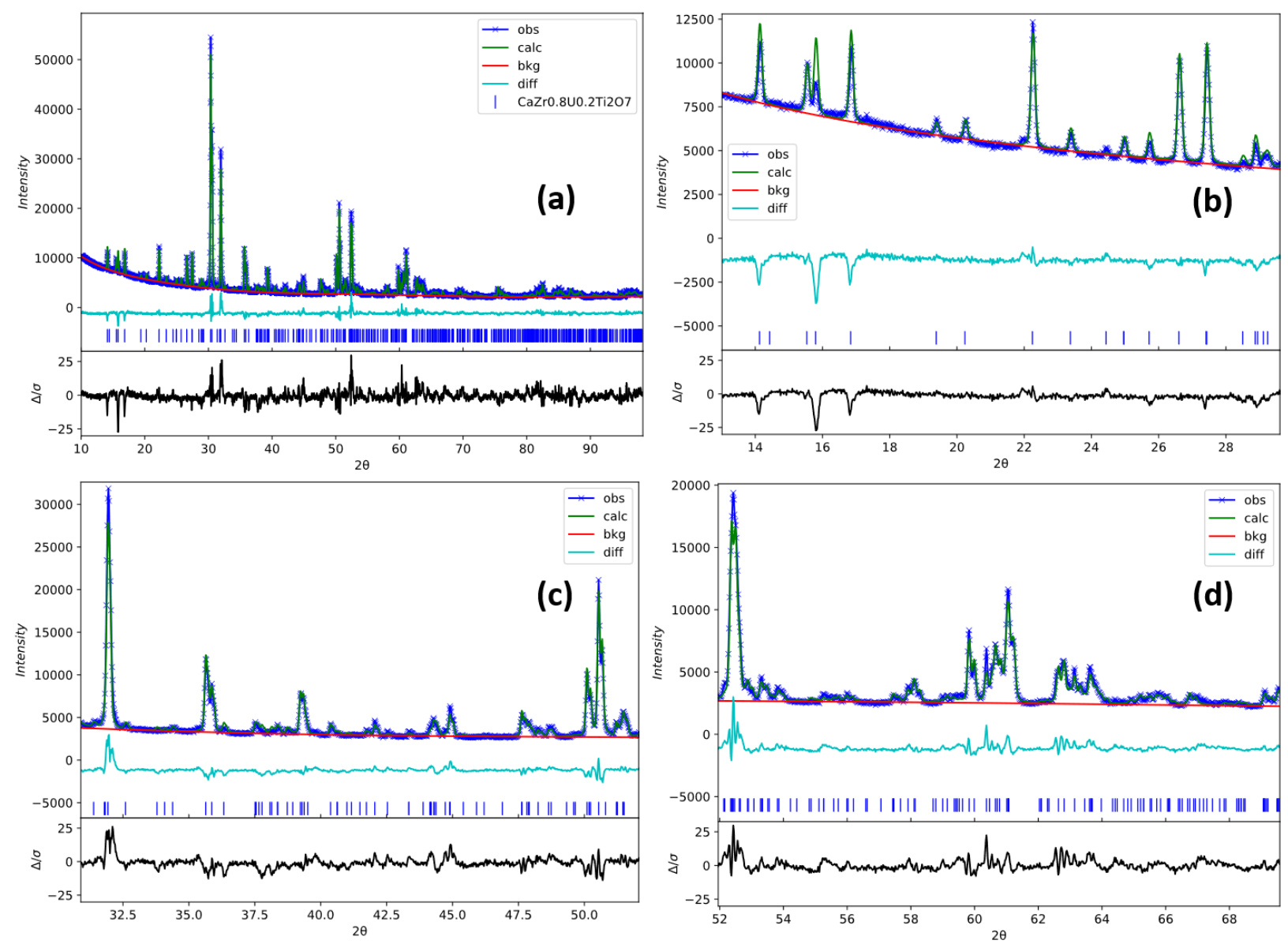

Figure S1 Rietveld refinement profile of $\mathrm{CaZr}_{0.8} \mathrm{U}_{0.2} \mathrm{Ti}_{2} \mathrm{O}_{7}$ (a) Full profile (b), (c) and (d) show refinement fitting of reflections between $2 \theta=13$ to $30^{\circ}, 32$ to $48^{\circ}$ and $2 \theta=48$ to $70^{\circ}$, respectively. 

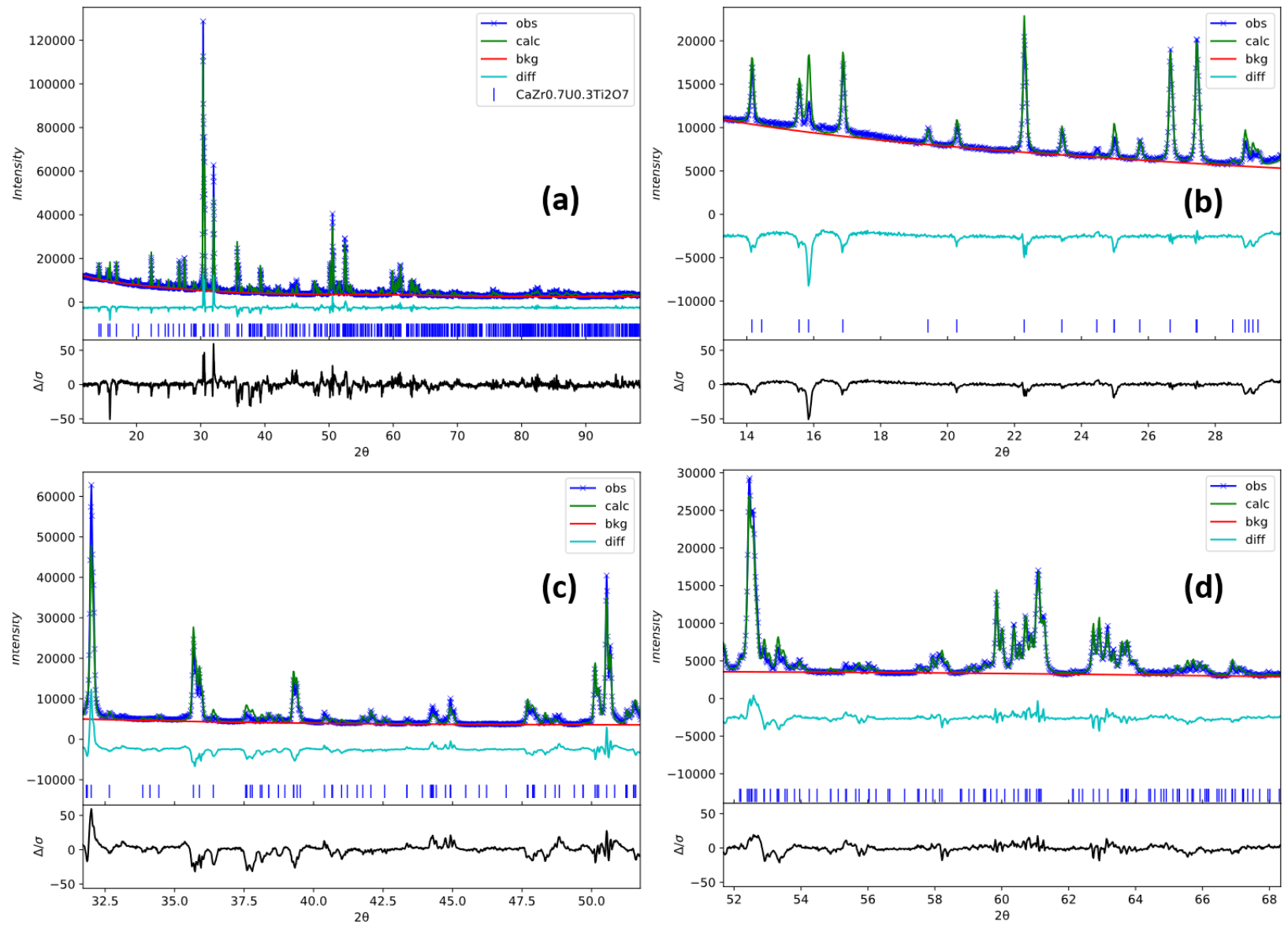

Figure S2 Rietveld refinement profile of $\mathrm{CaZr}_{0.7} \mathrm{U}_{0.3} \mathrm{Ti}_{2} \mathrm{O}_{7}$ (a) Full profile (b), (c) and (d) show refinement fitting of reflections between $2 \theta=13$ to $30^{\circ}, 32$ to $48^{\circ}$ and $2 \theta=48$ to $70^{\circ}$, respectively. 

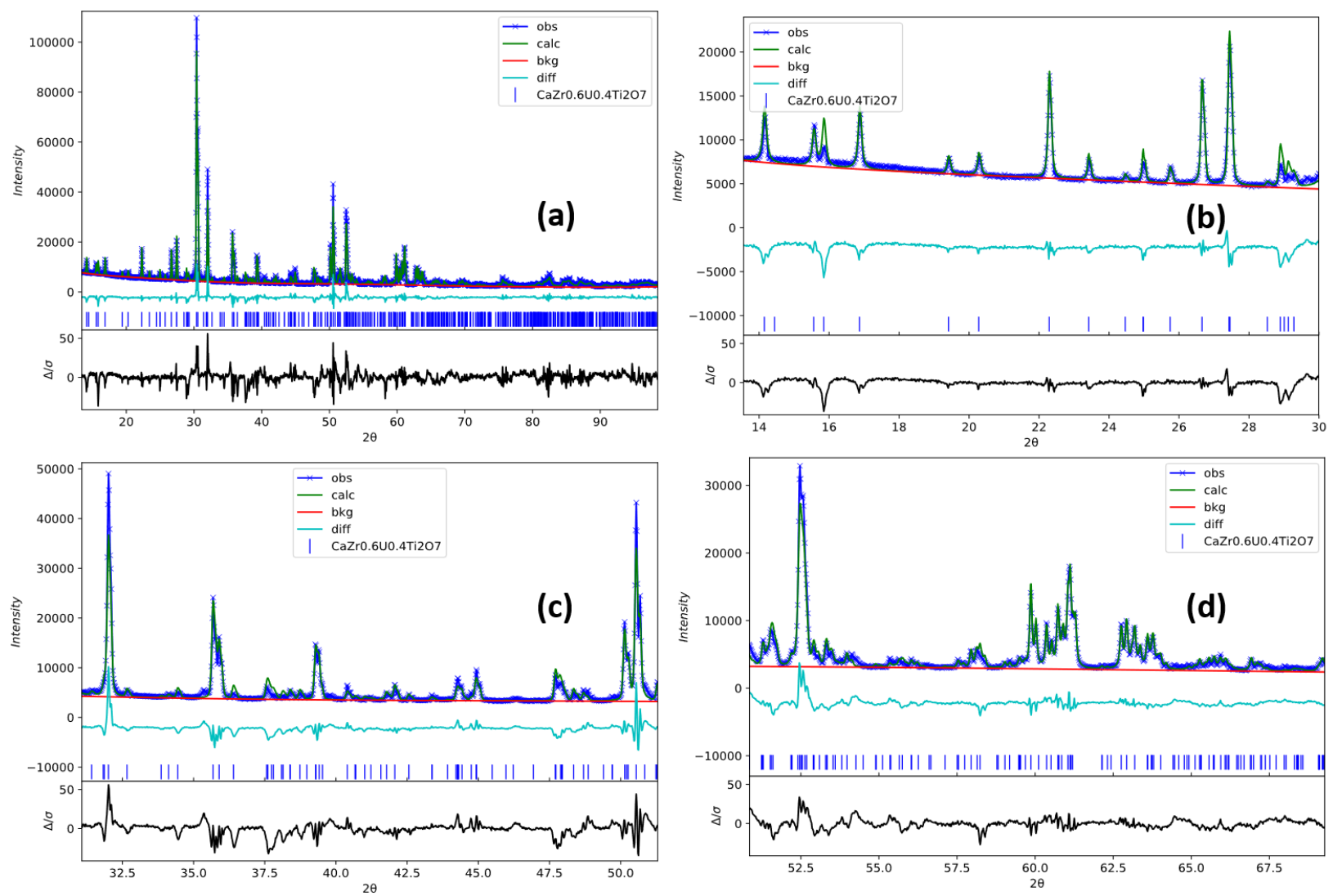

Figure S3 Rietveld refinement profile of $\mathrm{CaZr}_{0.6} \mathrm{U}_{0.4} \mathrm{Ti}_{2} \mathrm{O}_{7}$ (a) Full profile (b), (c) and (d) show refinement fitting of reflections between $2 \theta=13$ to $30^{\circ}, 32$ to $48^{\circ}$ and $2 \theta=48$ to $70^{\circ}$, respectively. 
Table S1 Crystallographic information of $\mathrm{CaZr}_{0.9} \mathrm{U}_{0.1} \mathrm{Ti}_{2} \mathrm{O}_{7.1}$ obtained from Rietveld analysis of PXRD patterns

\begin{tabular}{lllllll}
\hline Atom & Wycoff & $\mathbf{x}$ & $\mathbf{y}$ & $\mathbf{z}$ & occupancy & $\mathbf{U}_{\text {iso }}$ \\
\hline $\mathrm{Ca}$ & $8 f$ & $0.373(1)$ & $0.121(2)$ & $0.497(1)$ & 1.00 & $0.005(1)$ \\
$\mathrm{Zr} / \mathrm{U}$ & $8 f$ & $0.123(1)$ & $0.125(1)$ & $0.975(1)$ & $0.90 / 0.10$ & $0.011(1)$ \\
$\mathrm{Ti} 1$ & $8 f$ & $0.250(1)$ & $0.135(2)$ & $0.743(1)$ & 1.00 & $0.010(2)$ \\
$\mathrm{Ti} 2$ & $8 f$ & $0.466(1)$ & $0.064(2)$ & $0.258(2)$ & 0.50 & $0.038(6)$ \\
$\mathrm{Ti} 3$ & $4 e$ & 0 & $0.139(2)$ & 0.250 & 1.00 & $0.006(3)$ \\
$\mathrm{O} 1$ & $8 f$ & $0.298(1)$ & $0.127(4)$ & $0.283(1)$ & 1.00 & $-0.014(5)$ \\
O2 & $8 f$ & $0.474(2)$ & $0.125(5)$ & $0.101(2)$ & 1.00 & $0.039(8)$ \\
O3 & $8 f$ & $0.208(2)$ & $0.112(5)$ & $0.569(2)$ & 1.00 & $0.014(7)$ \\
O4 & $8 f$ & $0.390(2)$ & $0.137(4)$ & $0.727(2)$ & 1.00 & $0.007(5)$ \\
O5 & $8 f$ & $0.696(2)$ & $0.167(3)$ & $0.567(2)$ & 1.00 & $0.015(8)$ \\
O6 & $8 f$ & $-0.002(2)$ & $0.123(4)$ & $0.422(2)$ & 1.00 & $0.004(5)$ \\
O7 & $8 f$ & $0.112(2)$ & $0.041(3)$ & $0.727(2)$ & 1.00 & $0.007(5)$ \\
\hline
\end{tabular}

Unit cell parameters: $\mathrm{a}=12.463(1) \AA, \mathrm{b}=7.280(1) \AA, \mathrm{c}=11.405(1) \AA, \beta=$ $100.57(1)^{\circ}$

Unit cell volume $=1017.15(1) \AA^{3}$

Reliability factors: $\mathrm{R}=3.87 \%, \mathrm{wR}=5.46 \%, \mathrm{GOF}=4.31$ 
Table S2 Crystallographic information of $\mathrm{CaZr}_{0.8} \mathrm{U}_{0.2} \mathrm{Ti}_{2} \mathrm{O}_{7.2}$ obtained from Rietveld analysis of PXRD patterns

\begin{tabular}{|c|c|c|c|c|c|c|}
\hline Atom & Wycoff & $\mathrm{x}$ & $\mathrm{y}$ & $\mathrm{Z}$ & occupancy & $\mathrm{U}_{\text {iso }}$ \\
\hline $\mathrm{Ca}$ & $8 f$ & $0.379(1)$ & $0.118(2)$ & $0.502(1)$ & 1.00 & $0.020(2)$ \\
\hline $\mathrm{Zr} / \mathrm{U}$ & $8 f$ & $0.124(1)$ & $0.124(1)$ & $0.978(3)$ & $0.80 / 0.20$ & $0.038(1)$ \\
\hline Til & $8 f$ & $0.250(1)$ & $0.141(1)$ & $0.747(1)$ & 1.00 & $0.005(2)$ \\
\hline Ti2 & $8 f$ & $0.467(1)$ & $0.054(2)$ & $0.257(2)$ & 0.50 & $0.024(5)$ \\
\hline Ti3 & $4 e$ & 0 & $0.138(2)$ & 0.250 & 1.00 & $0.022(2)$ \\
\hline O1 & $8 f$ & $0.297(1)$ & $0.095(2)$ & $0.284(1)$ & 1.00 & $-0.018(6)$ \\
\hline $\mathrm{O} 2$ & $8 f$ & $0.475(2)$ & $0.134(3)$ & $0.101(1)$ & 1.00 & $0.002(6)$ \\
\hline $\mathrm{O} 3$ & $8 f$ & $0.208(2)$ & $0.122(3)$ & $0.560(1)$ & 1.00 & $0.011(5)$ \\
\hline $\mathrm{O} 4$ & $8 f$ & $0.387(1)$ & $0.145(3)$ & $0.721(1)$ & 1.00 & $0.007(5)$ \\
\hline $\mathrm{O} 5$ & $8 f$ & $0.709(2)$ & $0.171(3)$ & $0.589(2)$ & 1.00 & $0.005(7)$ \\
\hline O6 & $8 f$ & $0.016(2)$ & $0.153(4)$ & $0.436(2)$ & 1.00 & $0.045(8)$ \\
\hline O7 & $8 f$ & $0.088(2)$ & $0.042(2)$ & $0.771(2)$ & 1.00 & $0.016(7)$ \\
\hline \multicolumn{7}{|c|}{$\begin{array}{l}\text { Unit cell parameters: } \mathrm{a}=12.476(1) \AA, \mathrm{b}=7.281(1) \AA, \mathrm{c}=11.400(1) \AA, \beta= \\
100.51(1)^{\mathrm{o}} \\
\text { Unit cell volume }=1017.83(6) \AA^{3} \\
\text { Reliability factors: } \mathrm{R}=4.11 \%, \mathrm{wR}=5.69 \%, \mathrm{GOF}=3.67\end{array}$} \\
\hline
\end{tabular}


Table S3 Crystallographic information of $\mathrm{CaZr}_{0.7} \mathrm{U}_{0.3} \mathrm{Ti}_{2} \mathrm{O}_{7.3}$ obtained from Rietveld analysis of PXRD patterns

\begin{tabular}{|c|c|c|c|c|c|c|}
\hline Atom & Wycoff & $\mathrm{x}$ & $\mathrm{y}$ & $\mathrm{z}$ & occupancy & $\mathrm{U}_{\text {iso }}$ \\
\hline $\mathrm{Ca}$ & $8 f$ & $0.378(1)$ & $0.117(1)$ & $0.505(1)$ & 1.00 & $0.009(2)$ \\
\hline $\mathrm{Zr} / \mathrm{U}$ & $8 f$ & $0.126(4)$ & $0.130(1)$ & $0.980(1)$ & $0.70 / 0.30$ & $0.032(1)$ \\
\hline Ti1 & $8 f$ & $0.250(1)$ & $0.140(1)$ & $0.738(1)$ & 1.00 & $0.016(2)$ \\
\hline Ti2 & $8 f$ & $0.476(2)$ & $0.068(2)$ & $0.250(3)$ & 0.50 & $0.025(7)$ \\
\hline Ti3 & $4 e$ & 0 & $0.146(2)$ & 0.250 & 1.00 & $0.015(3)$ \\
\hline $\mathrm{O} 1$ & $8 f$ & $0.305(1)$ & $0.907(2)$ & $0.279(1)$ & 1.00 & $0.025(6)$ \\
\hline $\mathrm{O} 2$ & $8 f$ & $0.480(2)$ & $0.121(4)$ & $0.109(1)$ & 1.00 & $0.009(7)$ \\
\hline $\mathrm{O} 3$ & $8 f$ & $0.204(2)$ & $0.130(5)$ & $0.559(2)$ & 1.00 & $0.030(5)$ \\
\hline $\mathrm{O} 4$ & $8 f$ & $0.381(1)$ & $0.137(3)$ & $0.712(1)$ & 1.00 & $0.022(6)$ \\
\hline $\mathrm{O} 5$ & $8 f$ & $0.715(2)$ & $0.128(5)$ & $0.619(2)$ & 1.00 & $0.020(10)$ \\
\hline O6 & $8 f$ & $0.010(2)$ & $0.144(4)$ & $0.425(2)$ & 1.00 & $0.020(8)$ \\
\hline $\mathrm{O} 7$ & $8 f$ & $0.090(2)$ & $0.025(2)$ & $0.774(2)$ & 1.00 & $0.023(6)$ \\
\hline \multicolumn{7}{|c|}{$\begin{array}{l}\text { Unit cell parameters: } \mathrm{a}=12.473(1) \AA, \mathrm{b}=7.276(1) \AA, \mathrm{c}=11.3771) \AA, \beta= \\
100.60(1)^{\circ} \\
\text { Unit cell volume }=1014.82(5) \AA^{3} \\
\text { Reliability factors: } \mathrm{R}=5.29 \%, \mathrm{wR}=7.75 \%, \mathrm{GOF}=5.92\end{array}$} \\
\hline
\end{tabular}


Table S4 Crystallographic information of $\mathrm{CaZr}_{0.6} \mathrm{U}_{0.4} \mathrm{Ti}_{2} \mathrm{O}_{7.4}$ obtained from Rietveld analysis of PXRD patterns

\begin{tabular}{|c|c|c|c|c|c|c|}
\hline Atom & Wycoff & $\mathrm{X}$ & $\mathrm{y}$ & $\mathrm{Z}$ & occupancy & $\mathrm{U}_{\text {iso }}$ \\
\hline $\mathrm{Ca}$ & $8 f$ & $0.377(1)$ & $0.127(2)$ & $0.502(1)$ & 1.00 & $0.005(2)$ \\
\hline $\mathrm{Zr} / \mathrm{U}$ & $8 f$ & $0.124(1)$ & $0.130(1)$ & $0.977(1)$ & $0.60 / 0.40$ & $0.021(2)$ \\
\hline Ti1 & $8 f$ & $0.249(1)$ & $0.137(2)$ & $0.740(1)$ & 1.00 & $0.012(1)$ \\
\hline Ti2 & $8 f$ & $0.467(1)$ & $0.062(2)$ & $0.258(2)$ & 0.50 & $0.008(4)$ \\
\hline Ti3 & $4 e$ & 0 & $0.132(2)$ & 0.250 & 1.00 & $0.017(6)$ \\
\hline $\mathrm{O} 1$ & $8 f$ & $0.306(1)$ & $0.092(3)$ & $0.276(2)$ & 1.00 & $0.029(4)$ \\
\hline $\mathrm{O} 2$ & $8 f$ & $0.479(2)$ & $0.111(4)$ & $0.112(2)$ & 1.00 & $0.001(1)$ \\
\hline $\mathrm{O} 3$ & $8 f$ & $0.212(2)$ & $0.104(3)$ & $0.566(2)$ & 1.00 & $0.021(10)$ \\
\hline $\mathrm{O} 4$ & $8 f$ & $0.382(2)$ & $0.147(3)$ & $0.709(2)$ & 1.00 & $0.027(6)$ \\
\hline $\mathrm{O} 5$ & $8 f$ & $0.709(3)$ & $0.127(6)$ & $0.616(3)$ & 1.00 & $0.022(3)$ \\
\hline O6 & $8 f$ & $0.007(2)$ & $0.139(4)$ & $0.415(2)$ & 1.00 & $0.019(7)$ \\
\hline $\mathrm{O} 7$ & $8 f$ & $0.081(2)$ & $0.022(3)$ & $0.783(2)$ & 1.00 & $0.018(10)$ \\
\hline \multicolumn{7}{|c|}{$\begin{array}{l}\text { Unit cell parameters: } \mathrm{a}=12.476(1) \AA, \mathrm{b}=7.276(1) \AA, \mathrm{c}=11.377(1) \AA, \beta= \\
100.57(1)^{\circ} \\
\text { Unit cell volume }=1015.42(24) \AA^{3} \\
\text { Reliability factors: } \mathrm{R}=6.43 \%, \mathrm{wR}=8.94 \%, \mathrm{GOF}=6.37\end{array}$} \\
\hline
\end{tabular}


Table S5 Reliability factors and goodness of fit obtained from Rietveld refinements considering $\mathrm{U}$ in $\mathrm{Ti}$ positions in $\mathrm{CaZr}_{1-\mathrm{x}} \mathrm{U}_{\mathrm{x}} \mathrm{Ti}_{2} \mathrm{O}_{7+\mathrm{x}}$

\begin{tabular}{llll}
\hline Compound & R (\%) & wR $(\%)$ & GOF \\
\hline U in Ti1 (8f) site & & & \\
\hline $\mathrm{X}=0.1$ & 4.78 & 6.91 & 5.45 \\
$\mathrm{X}=0.2$ & 5.08 & 7.30 & 4.70 \\
$\mathrm{X}=0.3$ & 7.44 & 10.72 & 8.21 \\
$\mathrm{X}=0.4$ & 8.31 & 12.17 & 8.71 \\
\hline $\mathbf{U}$ in Ti3 (4e) site & & \\
\hline $\mathrm{X}=0.1$ & 5.02 & 7.17 & 5.66 \\
$\mathrm{X}=0.2$ & 7.08 & 10.33 & 6.65 \\
$\mathrm{X}=0.3$ & 7.46 & 11.18 & 8.56 \\
$\mathrm{X}=0.4$ & 7.79 & 11.59 & 8.29 \\
\hline
\end{tabular}


Table S6 Reliability factors and goodness of fit obtained from Rietveld refinements considering vacancies in $\mathrm{Ti}$ positions in $\mathrm{CaZr}_{1-\mathrm{x}} \mathrm{U}_{\mathrm{x}} \mathrm{Ti}_{2} \mathrm{O}_{7+\mathrm{x}}$

\begin{tabular}{|c|c|c|c|}
\hline Compound & $\mathrm{R}(\%)$ & wR (\%) & GOF \\
\hline \multicolumn{4}{|c|}{ Vacancies in Ti1 $(8 f)$ site } \\
\hline $\mathrm{x}=0.1$ & 4.03 & 5.67 & 4.48 \\
\hline $\mathrm{x}=0.2$ & 4.55 & 6.42 & 4.14 \\
\hline$x=0.3$ & 6.47 & 9.78 & 7.49 \\
\hline$x=0.4$ & 7.46 & 10.42 & 7.46 \\
\hline \multicolumn{4}{|c|}{ Vacancies in Ti2 $(8 f)$ site } \\
\hline $\mathrm{x}=0.1$ & 4.03 & 5.69 & 4.49 \\
\hline $\mathrm{x}=0.2$ & 4.80 & 7.24 & 4.64 \\
\hline$x=0.3$ & 6.45 & 9.84 & 7.54 \\
\hline $\mathrm{x}=0.4$ & 7.76 & 10.96 & 7.84 \\
\hline \multicolumn{4}{|c|}{ Vacancies in Ti3 (4e) site } \\
\hline $\mathrm{x}=0.1$ & 4.05 & 5.72 & 4.51 \\
\hline$x=0.2$ & 4.43 & 6.22 & 4.01 \\
\hline$x=0.3$ & 6.04 & 9.27 & 7.10 \\
\hline $\mathrm{x}=0.4$ & 6.67 & 9.47 & 6.77 \\
\hline \multicolumn{4}{|c|}{ Vacancies in all Ti sites } \\
\hline $\mathrm{x}=0.1$ & 3.98 & 5.62 & 4.43 \\
\hline$x=0.2$ & 4.54 & 6.54 & 4.27 \\
\hline$x=0.3$ & 5.95 & 9.07 & 6.94 \\
\hline$x=0.4$ & 6.94 & 9.89 & 7.07 \\
\hline
\end{tabular}

